\title{
LEFT VALUATION RINGS AND SIMPLE RADICAL RINGS(1)
}

\author{
BY \\ EDWARD C. POSNER
}

In this paper, we generalize the concept of valuation rings as defined in $[1$, Lemma 5, p. 9], and ask whether these left valuation rings have a unit. It happens that this question is intimately connected with the existence of certain kinds of simple radical rings; it is this connection which we wish to emphasize in this paper.

We define a left valuation ring to be a nonzero ring $R$ in which, for every $x, y$ in $R$, there exists a $u$ in $R$ such that either $x=u y$ or $y=u x$. A right valuation ring is defined similarly. (A valuation ring in the sense of [1] above is a left and right valuation ring, in the sense of this paper, without divisors of zero.)

There do exist left valuation rings which are not right valuation rings. An example can be given modifying $[2, \S 6$, p. 219]. The construction is, however, omitted.

We now prove some results valid for left valuation rings to lead up to the connection with simple radical rings. The lesser ones are called simply results, the more important ones theorems.

RESULT 1 . The left ideals of $R$ are linearly ordered by inclusion.

Proof. If $I_{1}$ and $I_{2}$ are left ideals of $R$, and $I_{1} \notin I_{2}$, we shall prove that $I_{2} \subset I_{1}$. Since $I_{1} \notin I_{2}$, there is an $x$ in $I_{1}$ but not in $I_{2}$. Let $y$ be an arbitrary element of $I_{2}$. If $x=u y, u \in R$, then $x$ is in $I_{2}$. Therefore, $y=u x, u \in R$, and $y$ is in $I_{1}$. Thus $I_{2} \subset I_{1}$, as required.

REMARK. If $R$ has a unit, then $R$ is a left valuation ring if and only if the left ideals of $R$ are linearly ordered by inclusion. But if $R$ lacks a unit, the two may be shown to be not equivalent. However, if $R$ is an algebra over a field $F$ of characteristic zero, and the (ring) ideals of $R$ are linearly ordered by inclusion, then the two concepts are equivalent; the proof is omitted.

Result 2. $R$ has no absolute right divisors of zero.

Proof. If $R x=0$, then, since $x=u x, u \in R$, we conclude that $x=0$, as required.

Result 3. $R$ is not a radical ring.

Proof. Let $x$ be a nonzero element of $R$, and let $x=u x, u \in R$, i.e., $(1-u) x=0$. (We use a dummy unit throughout this paper for such purposes even if $R$ lacks a unit.) Thus $1-u$ cannot have a left inverse $1-v, v \in R$, for then

Received by the editors December 10, 1961 .

(1) Done at the Jet Propulsion Laboratory of the California Institute of Technology with support of a NASA contract. 
$(1-v)[(1-u) x]=[(1-v)(1-u)] x=x=0$. Thus $u$ is not in the radical of $R$.

RESUlt 4. If $x \notin N$, the radical of $R$, then $R x=R$.

Proof. Let $M$ be a regular maximal left ideal of $R$ not containing $x$, which ideal exists since $x \notin N$. Assume $R x \neq R$. Then $R x \subset M$. For if $M \subset R x$, by Result 1 the only other possibility, since $M$ is maximal and $R x \neq R$, we would have $R x=M$. So in any case $R x \subset M$. Since $x \in R x, x \in M$. This contradiction proves that $R x=R$.

Result 5. If $x \notin N$, then $x$ is not a left zero divisor in $R$.

Proof. If $x \notin N$, then $R x=R$, by the preceding result. If $x y=0$, then $R y=(R x) y=R(x y)=R \cdot 0=0$. By Result 2, $y=0$, as required.

Result 6. $R / N$ is a division ring.

Proof. Let $x+N$ be a nonzero element of $R / N$; such exist by Result 3. Since $R x=R$ by Result 4, every element of $R / N$ is a left multiple of $x+N$. This proves that $R / N$ is a division ring.

ResUlt 7. If $R$ contains a left or right unit, then $R$ contains a unit. In fact, any nonzero idempotent of $R$ is a unit of $R$.

Proof. Let $e \in R, e^{2}=e, e \neq 0$. Then $e \notin N$, so $R=R e$ by Result 4. Thus $e$ is a right unit of $R$. Also $e(r-e r)=0$ for all $r$ in $R$. Since $e \notin N, r-e r=0$ by Result 5 . Thus $e$ is in fact a two-sided unit of $R$.

ReSUlt 8 . If $R$ is commutative, then $R$ has a unit. In fact, if $R$ is also a right valuation ring, then $R$ has a unit.

Since we shall not need this result, its proof is omitted. We remark that a proof can be given not using Zorn's lemma or the radical.

RESULT 9. If $U$ is a two-sided nil ideal modulo which $R$ has a unit, then $R$ itself has a unit.

Proof. Let $e+U$ be a unit of $R / U$, so that $e-e^{2} \in U$ and, in particular, $e-e^{2}$ is nilpotent. Let $\left(e-e^{2}\right)^{n}=0$; then $e^{n}(1-e)^{n}=0, e^{n}\left[(1-e)^{n} r\right]=0$ for all $r$ in $R$. Now $U \subset N$ since $U$ is nil; and, since $e$ is a unit for $R / U, r-r e \in U$ for all $r$ in $R$. If $e$ were in $N$, then $r-r e \in N, r e \in N$, and $r$ would be in $N$ for every $r$ in $R$. By Result 3, however, $R \neq N$; thus $e \notin N$. By Result $6, e^{n} \notin N$ either. By Result $5, e^{n}$ is not a left zero divisor in $R$. Thus $(1-e)^{n} r=0$ for all $r$ in $R$. In other words, $\sum_{i=1}^{n}(-1)^{i+1} C_{n, i} e^{i}$ is a left unit for $R$, and, by Result 7 , a unit for $R$.

Result 10. If $R$ has no nilpotent ideals, then $R$ is a prime ring. That is, $R$ modulo its lower nil radical is a prime left valuation ring.

Proof. That any (nonzero) quotient ring of a left valuation ring is a left valuation ring is trivial; thus we may suppose that $R$ itself has no nilpotent ideals. Let $I, J$ be left ideals of $R$ with $I J=0$. By Result 1, either $I \subset J$ or $J \subset I$. If $I \subset J$, then $I^{2}=0$; if $J \subset I$, then $J^{2}=0$. Since $R$ has no nilpotent ideals, either $I=0$ or $J=0$ if $I J=0$. In other words, $R$ is prime. 
RESULT 11. Let $U$ be the lower nil radical of $R$. If the set of nilpotent elements of $R$ is not equal to $U$, then there is a smallest two-sided ideal $Q$ of $R$ containing $U$ properly, and $Q$ contains the left annihilator of every element of $R$ not in $U$ (and thus contains every nilpotent element of $R$ ).

Proof. The ring $R / U$ is also a left valuation ring. It is enough to prove the result for $R / U$; thus, we may assume that $U=0$, i.e., that $R$ has no nilpotent ideals. Let $u, v$ be two nonzero elements of $R$ with $u v=0$. We shall prove that $u$ is in every nonzero two-sided ideal $K$ of $R$. If $u \notin K$, then $R u \notin K$, and, by Result 1 , $K \subset R u$. Then $K v \subset R u v=0, K v=0$. Since $R$ is prime by the preceding result, we conclude that $K=0$. This contradiction proves that $u \in K$. Let $Q$ be the intersection of the nonzero two-sided ideals of $R$. We have shown that $Q$ contains every left zero divisor of $R$. This proves the result.

THEOREM 1. If $R$ is a left valuation ring, and if the lower nil radical $U$ of $R$ fails to contain the left annihilator of every element of $R$ not in $U$, then the minimal two-sided ideal $Q$ of $R / U$ is a simple radical ring. (In particular, the hypotheses are satisfied if $R$ is a left valuation ring with no unit.)

Proof. If $R$ is a left valuation ring with no unit, then $R / U$ has no unit by Result 9. Thus $U$ cannot contain the left annihilator of every element of $R$ not in $U$, for if it did contain all such elements, then $R / U$ would have no divisors of zero, and a fortiori would have a unit.

So let $R$ be a left valuation ring whose lower nil radical $U$ fails to contain the left annihilator of every element of $R$ not in it. (The ideal $Q$ exists by the preceding result.) Let $q$ be a nonzero element of $Q$; we are to prove that $Q q Q=Q$. Observe that $Q q Q$ is a two-sided ideal of $R / U$ contained in $Q$. Since $Q$ is a minimal twosided ideal of $R / U, Q q Q=0$ or $Q q Q=Q$. But if $Q q Q=0$, then $(Q q)^{2}=0$, and $Q q=0$, since $R / U$ has no nilpotent ideals. But $R / U$ is prime by Result 10 , so, since $Q \neq 0, q$ would be zero. This contradiction proves that $Q q Q \neq 0$, so that $Q q Q=Q$. And by Result $1, Q \subset N$ or $N \subset Q$. But $N \subsetneq Q$ is not possible since $N \neq 0$ by Result 6 . Thus $N=Q$ or $Q \subset N$; we have therefore $Q \subset N$. But any ideal of the radical ring $N$ is a radical ring in its own right. This proves that $Q$ is a radical ring and completes the proof of Theorem 1 .

COROLLARY. If the left valuation ring $R$ is a polynomial identity algebra, then $R$ has a unit. Also, if $R$ is a left valuation ring with no nilpotent ideals and satisfies a polynomial identity with coefficients in the centroid of $R$, then $R$ has $a$ unit and no divisors of zero.

Proof. In the first case, it is enough, by Result 9 , to prove that $R / U$ has a unit, where $U$ is the lower nil radical of $R$. Now $U$ is in fact an algebra ideal of $R$, so that $R / U$ is a polynomial identity algebra (and, of course, a left valuation ring). 
In other words, we may assume that $R$ has no nilpotent ideals. We shall prove that the ideal $Q$ of $R$, which exists by Result 11 if $R$ has no unit or has zero divisors, is an algebra ideal of $R$. Let $\lambda$ be a field element, and $q \in Q$. Since $Q^{2}=Q, q$ can be written as $\sum_{i=1}^{n} q_{i} q^{(i)}, q_{i}, q^{(i)} \in Q, 1 \leqq i \leqq n$. Then $\lambda q=\sum_{i=1}^{n}\left(\lambda q_{i}\right) q^{(i)}$ is a sum of elements of $Q$, hence is in $Q$. Thus $Q$, being an algebra ideal of $R$, is a polynomial identity algebra, too. By Theorem $1, Q$ is a simple radical ring. These two facts taken together, however, are a direct contradiction to [3, $\$ 2$, Problem 2, p. 1]. This proves the corollary in the first case.

In the second case, $Q$ is invariant under the centroid of $R$ as above. Furthermore, since $R$ is prime by Result 10, no nonzero element of the centroid of $R$ has a kernel other than zero. Therefore the coefficients of the polynomial identity do not vanish on $Q$, so that $Q$ is a ring satisfying a polynomial identity over its centroid. Since $Q$ is a simple radical ring, we obtain the same contradiction as in the first case.

An alternative proof of this corollary can be obtained by using [4, Paragraph 3, p. 180], which states that a prime ring satisfying a polynomial identity over its centroid has the ascending chain condition on its left and right annihilator ideals. (As above, we may assume $R$ prime.) We may then directly apply Theorem 2 of this paper (see below).

RESULT 12. If $R$ is a left valuation ring and $u_{1} v_{1}=u_{2} v_{2}=0$, then $u_{1} v_{2}=0$ or $u_{2} v_{1}=0$.

Proof. Let $L_{1}$ be the left annihilator ideal of $v_{1}$ and $L_{2}$ of $v_{2}$. If, say, $L_{1} \subset L_{2}, u_{1} v_{2}=0$. If $L_{2} \subset L_{1}, u_{2} v_{1}=0$. This proves the result.

LEMMA 1. Let $J$ be a left (or right) ideal of a radical ring $A$ invariant under every automorphism of $A$ of the form $a \rightarrow\left(1+q^{\prime}\right) a(1+q), q \in A, q^{\prime}$ the quasiinverse of $q$. (Such an automorphism is called a quasi-inner automorphism.) Then $J$ is a two-sided ideal of $A$.

Proof. Let $J$ be, say, a left ideal of $A$. Let $q \in A, j \in J$. We shall prove that $j q \in J$, so that $J$ will also be a right ideal of $A$, hence a two-sided ideal of $A$. Consider the quasi-inner automorphism $a \rightarrow\left(1+q^{\prime}\right) a(1+q)$ of $A$. By hypothesis, $\left(1+q^{\prime}\right) j(1+q) \in J,\left(1+q^{\prime}\right) j+\left(1+q^{\prime}\right) j q \in J$. Since $J$ is a left ideal of $A,\left(1+q^{\prime}\right) j \in J$, so that $\left(1+q^{\prime}\right) j q \in J$. Then

$$
(1+q)\left[\left(1+q^{\prime}\right)\right] j q=\left[(1+q)\left(1+q^{\prime}\right)\right] j q=j q \in J
$$

as promised.

RESULT 13. If the lower nil radical $U$ is not the set of left annihilators of elements of $R$ not in $U$, then $Q$ (which exists by Result 11) is the set of left annihilators of elements of $R$ not in $U$.

Proof. Let $L$ denote the set of left annihilators of elements of $R$ not in $U$. The set $L$ is invariant under every automorphism of $R$, since $U$ is invariant under 
every automorphism of $R$. We shall prove that $L$ is a left ideal of $R$. Let $u_{1}, u_{2}$ be two elements of $L$; then there exist $v_{1}, v_{2}$ lin $R$ but not in $U$ such that $u_{1} v_{1}=u_{2} v_{2}=0$. By Result 12, either $u_{1} v_{2}=0$ or $u_{2} v_{1}=0$. Suppose $u_{1} v_{2}=0$. Then $\left(u_{1}-u_{2}\right) v_{2}=0$, so that $L$ is closed under subtraction. Since $R L \subset L$ trivially, $L$ is a left ideal of $R$. By Result 11, $L \subset Q$, so that $L$ is a left ideal of $Q$. Since every quasi-inner automorphism of $Q$ is the restriction to $Q$ of a quasiinner automorphism of $R, L$ is invariant under every quasi-inner automorphism of $Q$. Thus $L$ is a two-sided ideal of $Q$, by Lemma 1 . Since $Q / U$ is a simple ring by Theorem $1, L=U$ or $L=Q$. Since $L \neq U$ by hypothesis, $L=Q$, as required.

RESULT 14. $R / Q$ has no divisors of zero (and a unit).

Proof. We clearly may assume $U=0$. If $x, y$ are elements of $R$ with $x y \in Q$, then $x y$ is a left divisor of zero (or zero itself) by the preceding result. That is, $(x y) z=0$ for some nonzero $z$ in $R$. If $y z=0$, then $y$ is in $Q$ by Result 11 ; if $y z \neq 0$, then $x$ is in $Q$ by that same result. This proves Result 14 .

RESUlt 15. If some $x$ in $R$ but not in $U$ has a right unit, then $R$ itself has a unit.

Proof. Assume that $R$ lacks a unit but that $x=x u, x$ an element of $R$ not in $U$. Consider the set $P$ of elements of $R$ which have right units. As in the preceding result, we prove that $P$ is a left ideal of $R$, so that $P \subset Q$ or $Q \subset P$. If $P \subset Q$, we conclude that $P$ is a two-sided ideal of $Q$ as in the preceding result, and then similarly conclude that $P=Q$. Thus in any event, $Q \subset P$. Let $z$ belong to $R$ but not to $N$, the radical of $R$. Let $z=t z, t$ an element of $R$. Then $(z-z t) z=0$; since $z \notin N, z \notin U$, and $z-z l \in Q$ by Result 11 . Therefore, $z-z t \in P$, so that $z-z t=(z-z t) w$ for some $w$ in $R$. Thus $z=z(t+w-t w)$. Since $z \notin N$, $R=R z$ by Result 4. Thus $t+w-t w$ is a right unit for $R$, hence a unit by $R e-$ sult 7. This proves Result 15.

RESULT 16. If $R$ lacks a unit, then every nonzero element of $R$ is a right annihilator of an element of $R$ not in $U$. In any case, either $U$ is the set of right annihilators of elements of $R$, or else every element of $Q$ (which then exists by Result 11) is a right annihilator of an element of $R$ not in $U$.

Proof. In the first case, let $x \in R, x \notin U$, and let $x=u x, u$ an element of $R$. Then $(x-x u) x=0$; if $x-x u \in U$, then the previous result shows that $R$ has a unit. Thus $x-x u \notin U$, and $x$ is indeed a right annihilator in $R$ of an element not in $U$. This proves the first part of the result; the proof of the second part is similar to the proof of Result 13 and is omitted.

RESULT 17. If $L$ is a left ideal of $R$, and $q$ is a quasi-regular element of $R$ with quasi-inverse $q^{\prime}$, then either $L q \subset L$ or $L q^{\prime} \subset L$. If $q$ is nilpotent, $L q \nsubseteq L$ (unless $L=0$ ).

Proof. Let $\sigma$ denote the quasi-inner automorphism of $R$ defined by $\sigma(a)=\left(1+q^{\prime}\right) a(1+q)$. Since $\sigma(L)$ is a left ideal of $R$, either $\sigma(L) \subset L$ or $L \subset \sigma(L)$. Say $\sigma(L) \subset L$. Thus if $t \in L,\left(1+q^{\prime}\right) t(1+q)=t_{1} \in L$, $(1+q)\left[\left(1+q^{\prime}\right) t(1+q)\right]=t(1+q)=(1+q) t_{1}=t_{2} \in L, t q=t_{2}-t \in L$. We 
have shown that $L q \subset L$ if $L \subset \sigma(L)$. Similarly, $L q^{\prime} \subset L$ if $\sigma(L) \subset L$. If $q$ is nilpotent, with $q^{n}=0$, then $\left(q^{\prime}\right)^{n}=0$, and

$$
q\left(=\left(q^{\prime}\right)^{\prime}\right)=-q^{\prime}+\left(q^{\prime}\right)^{2}-\left(q^{\prime}\right)^{3}+\cdots+(-1)^{n-1}\left(q^{\prime}\right)^{n-1} .
$$

Thus if $L q^{\prime} \subset L$, then $L q \subset L$, too, so that $L q \subset L$ in any event. If $L=L q$, then $L=L q^{n}=0$. This completes the proof.

RESUlt 18. If $u v=0$ and $t$ is nilpotent, then $u t v=0$.

Proof. Let $L$ denote the left ideal of left annihilators of $v$. By the previous result, $L t \subset L$. Thus $u t \in L$ if $u \in L$, or $u t v=0$ if $u v=0$.

Definition. A ring $A$ is called zero-divisor expandable if, whenever $u, v, t \in A$ and $u v=0$, then $u t v=0$. (Thus any commutative ring, as well as any ring without zero divisors, is zero-divisor expandable.)

RESUlt 19. The set $A$ of nilpotent elements of $R$ form a zero-divisor expandable nil ring.

Proof. By Result 18, we need only prove that $A$ is a subring of $R$. Let $x, y \in A$, with $x^{m}=y^{n}=0$, and let $p=\max (m, n)$. Then $(x y)^{p}=0$ by successive applications of Result 18, using $x$ as $t$ each time if $p=n$, and $y$ as $t$ if $p=m$. Thus $A$ is closed under multiplication. We can thereupon prove that $(x-y)^{m+n-1}=0$, for every term in this expansion has $m$ factors of $x$ or $n$ factors of $y$. Thus $A$ is also closed under subtraction and hence is indeed a subring of $R$.

RESULT 20. Let $R$ be a left valuation ring with no nilpotent ideals which has divisors of zero. Then the minimal ideal $Q$ of $R$ is not nil.

Proof. If $Q$ were nil, then $Q$ would be zero-divisor expandable by the previous result. Now $Q$ is simple, hence prime; we prove that a prime zero-divisor expandable ring $Q$ has no divisors of zero. Let $u, v \in Q$ with $u v=0$; by the definition of zerodivisor expandability, $u Q v=0$. Since $Q$ is prime, $u=0$ or $v=0$, as promised. Thus $Q$ has no zero divisors, hence is not nil. This proves the result.

Result 21. The upper nil radical $T$ of a left valuation ring $R$ coincides with the lower nil radical $U$ of $R$ and contains every one-sided nil ideal of $R$.

Proof. This amounts to proving that if $R$ has no nilpotent ideals, then it has no nil one-sided ideals. Let $R$ have no nilpotent ideals, and let $B$ denote the sum of the nil left ideals of $R$. Since the nil left ideals of $R$ are linearly ordered by inclusion, $B$ is nil. Since an element $x$ of any ring lies in a nil left ideal if and only if it lies in a nil right ideal, $B$ is also the sum of the nil right ideals of $R$. In summary, $B$ is the upper nil radical $T$ of $R$, and $T$ contains every nil one-sided ideal of $R$.

By Result 11, if $T \neq 0$, then $R$ has a minimal two-sided ideal $Q$, and $T \subset Q$. By the previous result, $T \neq Q$. Thus $T=0$ if $T \neq 0$; this contradiction proves the result.

RESULT 22. Let $R$ have divisors of zero, but let $U$ not be the set of left zero divisors of elements of $R$ not in $U$. Define $K=\{q v / q \in Q, v$ nilpotent $\}$. Then $K=Q$. (In particular, $K=Q$ if $R$ has no unit.) 
Proof. Observe that $K \subset Q$. Also, $K$ is invariant under any automorphism of $R$. For $Q$ is invariant under any automorphism of $R$, since $Q$ is precisely the set of left annihilators of elements of $R$ not in $U$, by Result 13. We shall show that $K$ is a left ideal of $R$. It is trivial that $R K \subset K$; we shall prove that $K$ is closed under subtraction. Let $v_{1}^{m}=0, v_{2}^{n}=0$, and suppose $Q v_{1} \subset Q v_{2}$. Then if $q_{1}, q_{2} \in Q$, $q_{1} v_{1} \in Q v_{2}$, and $q_{1} v_{1}-q_{2} v_{2} \in Q v_{2}$. This proves that $K$ is closed under subtraction and thus is a left ideal of $R$, hence of $Q$. By Lemma $1, K$ is a two-sided ideal of $Q$. (We may assume that $U=0$, for $R / U$ is prime by Result 10 , and any prime ring with divisors of zero has nilpotent elements.) Let $v^{n}=0, v \neq 0$. Since $v \neq 0$ and $R$ is prime, $Q v \neq 0$. Thus $K \neq 0$, and since $Q$ is simple, $K=Q$ as required.

RESULT 23. Let $R$ have no unit, or let $R$ have no nilpotent ideals but have divisors of zero. Then the nil subring $A$ of $R$ is not nilpotent.

Proof. We may assume that $R$ has no nilpotent ideals, for if $U$ is the nil radical of $R$ (here we are using Result 21 to call $U$ the nil radical) and $A_{U}$ is the nil radical of $R / U$, then $A_{U}=A / U$. Thus if $A$ is nilpotent, so is $A_{U} ; R / U$ lacks a unit if $R$ lacks a unit, by Result 9. Thus we assume that $R$ has no nilpotent ideals. Since $R$ lacks a unit, or has divisors of zero, $R$ has a minimal two-sided ideal $Q$, and $Q=\{q v / q \in Q, v \in A\}$, by the previous result. Let $q$ be an arbitrary element of $Q$; we shall show that $q=0$ and thus obtain the contradiction $Q=0$.

Since $q \in Q, q=q_{1} v_{1}, q_{1} \in Q, v_{1} \in A$. Also $q_{1}=q_{2} v_{2}, q_{2} \in Q, v_{2} \in A$. Continuing in this way, we find that $q=q_{n} v_{n} v_{n-1} \cdots v_{2} v_{1}, q_{n} \in Q, v_{i} \in A, 1 \leqq i \leqq n$, for all positive integers $n$. If now $n$ is such that $A^{n}=0$, then $v_{n} v_{n-1} \cdots v_{2} v_{1}=0$, and $q=0$, as required. (We have, in fact, proven that $A$ is not sequentially nilpotent; a ring $A$ is called sequentially nilpotent if every sequence $v_{1}, v_{2} v_{1}, \cdots v_{n} v_{n-1} \cdots v_{2} v_{1}, \cdots$, of elements of $A$ with each $v_{i}$ in $A$ contains zero.)

THEOREM 2. If the left valuation ring $R$ has the maximum or minimum condition on either left or right annihilator ideals, then $R$ has a unit and $U$ is the set of nilpotent elements of $R$. If the left valuation ring $R$ has no nilpotent ideals and has a maximum or minimum left or right annihilator ideal, then $R$ has a unit and no divisors of zero.

Proof. Since $R / U$ satisfies the same chain conditions that $R$ satisfies, we may, by Result 9, assume that $U=0$. We are then to prove that $R$ has no divisors of zero. Since $R$ has a minimum left annihilator ideal if and only if $R$ has a maximum right annihilator ideal, and a maximum left annihilator ideal if and only if it has a minimum right annihilator ideal, we need only treat the cases in which $R$ has a minimum left annihilator ideal and a maximum left annihilator ideal, assuming in both cases that $R$ has divisors of zero but no nilpotent ideals.

First let $R$ have a minimum left annihilator ideal $I$. Since the left ideals of $R$ are linearly ordered by inclusion, $I$ is contained in every left annihilator ideal of $R$. But by Result 16, every element of the ideal $Q$, which exists by Result 11 , is a right 
zero divisor in $R$. In other words, every element $q$ in $Q$ belongs to a left annihilator ideal $I^{(q)}$ of $R$. And $I \subset I^{(q)}$, so that $I q=0$ for every $q$ in $Q$. Thus $I Q=0$. Since $R$ is prime by Result 10 , we obtain the contradiction $I=0$ or $Q=0$. This completes the proof in case $R$ has a minimum left annihilator ideal.

Now suppose that $R$ has a maximum left annihilator ideal $I$. By Result 11, $I \subset Q$. Since every element of $Q$ is a left divisor of zero by Result 13 , every $q$ in $Q$ belongs to a left annihilator ideal $I^{(q)}$, which must be contained in $I$ by hypothesis. Thus $Q \subset I$, and $Q=I$. Since $I$ is a left annihilator ideal of $R, I v=0$ for some nonzero $v$ in $R$. Thus $Q v=0$, which is a contradiction since $R$ is prime. This completes the proof of Theorem 2. (An alternative proof of Theorem 2, assuming, however, that $R$ satisfies the ascending chain condition on either left or right ideals, can be given by combining Result 23 , which states that the nil subring $A$ of $R$ is not nilpotent, with [2, Theorem 6.1, p. 220], which states the contrary.)

In conclusion, we conjecture that there exists a left valuation ring without a unit and thus that there exists a simple radical ring with zero divisors whose set of nilpotent elements form a zero-divisor expandable ring. (This would also provide a negative solution to [6, Problem 8 , (a) and (b), p. 529].)

\section{BIBLIOGRAPHY}

1. O. F. G. Schilling, The theory of valuations, Math Surveys No. 4, Amer. Math. Soc., Providence, R. I. 1950.

2. A. W. Goldie, Semi-prime with maximum condition, Proc. London Math. Soc. 10 (1960), 201-220.

3. I. Kaplansky, Linear algebra, pp. 1-3, National Research Council, Publ.502, National Academy of Sciences, Washington, D. C., 1957.

4. E. C. Posner, Prime rings satisfying a polynomial identity, Proc. Amer. Math. Soc, 11 (1960), 180-183.

5. N. Jacobson, Structure of rings, Amer. Math. Soc. Colloq. Publ. Vol. 37, Amer. Math. Soc., Providence, R. I., 1956.

6. I. N. Herstein, Lie and Jordan structures in simple, associative rings, Bull. Amer. Math. Soc. 67 (1961), 517-531.

California Institute of Technology, Pasadena, California 